\title{
The Effects of Pharmacological Interventions on Smoking Cessation in People with Alcohol Dependence: A Systematic Review and Meta-analysis of Nine Randomized Controlled Trials
}

\author{
kangle Guo ${ }^{1}$, Jingwen $\mathrm{Li}^{1}$, Li Jieyun ${ }^{1}$, Nan Chen ${ }^{1}$, yanfei li ${ }^{1}$, kehu yang ${ }^{1}$, and Xiuxia $\mathrm{Li}^{1}$ \\ ${ }^{1}$ Lanzhou University
}

February 19, 2021

\begin{abstract}
Background Pharmacotherapies are widely used for smoking cessation. However, their effect on smoking cessation for people with alcohol dependence remains unclear. Objective This study aimed to explore the effects of pharmacotherapies on smoking cessation for people with alcohol dependence. Methods Five electronic databases were searched in January 2021 for randomized controlled trials (RCTs) reporting the use of pharmacotherapies to promote smoking cessation in people with alcohol dependence. The risk of bias was assessed using the Cochrane tool. RevMan version 5.3 was used to perform meta-analyses of the changes in smoking behavior, and the GRADE approach was used to assess the certainty of the evidence. Results Nine RCTs involving 908 smokers with alcohol dependence were identified. Eight RCTs were published in the United States, and one was from Canada. The risk of bias was rated as low in three studies and unclear in the remaining six. The results of the meta-analysis showed that, compared with the placebo group, Varenicline had a significant effect on short-term smoking cessation (three RCTs, OR $=6.27$, 95\% CI: $[2.49,15.78], \mathrm{p}<0.05$, very low certainty). Naltrexone had no significant effect on smoking cessation in short-term or long-term observations (three RCTs, OR $=0.99,95 \% \mathrm{CI}$ : $[0.54,1.81], \mathrm{p}=0.97$, moderate certainty), and Topiramate had no significant effect (two RCTs, OR $=1.56,95 \% \mathrm{CI}$ : $[0.67,3.46], \mathrm{p}>0.05$, low certainty). Only one trial reported that Bupropion had no effect on smoking cessation. Conclusion Varenicline may have a positive effect on smoking cessation in people with alcohol dependence. However, Naltrexone, Topiramate, and Bupropion seem to have no clear effect on increasing smoking abstinence among drinkers. The small number of studies and the low certainty of evidence indicate that caution is required in interpreting the results.
\end{abstract}

The Effects of Pharmacological Interventions on Smoking Cessation in People with Alcohol Dependence: A Systematic Review and Meta-analysis of Nine Randomized Controlled Trials Kangle Guo ${ }^{1,2,3 \#}$, Jingwen, $\mathrm{Li}^{1,2 \#}$, Jieyun $\mathrm{Li}^{2}$, Nan Chen ${ }^{1,2}$, Yanfei $\mathrm{Li}^{1,2}$, Kehu Yang ${ }^{1,2,3^{*}}$, Xiuxia Li ${ }^{1,2,3^{*}}$ ${ }^{1}$ Health Technology Assessment Center/Evidence-Based Social Science Research Center, School of Public Health, Lanzhou University, Lanzhou, China

${ }^{2}$ Evidence Based Medicine Center, School of Basic Medical Sciences, Lanzhou University, Lanzhou, China

${ }^{3}$ Key Laboratory of Evidence Based Medicine and Knowledge Translation of Gansu Province, Lanzhou, 730000, China

\# Kangle Guo and Jinwen Li are co-first authors.

*Correspondence authors:

Kehu Yang 
Address: Evidence Based Medicine Center, School of Basic Medical Sciences, Lanzhou University, 199 Donggang West Road, Lanzhou 730000, China.

Emails: yangkh-ebm@lzu.edu.cn

Xiuxia Li

Address: Health Technology Assessment Center/Evidence-Based Social Science Research Center, School of Public Health, Lanzhou University, 199 Donggang West Road, Lanzhou 730000, China.

Emails: lixiuxia@lzu.edu.cn

\section{Declarations:}

Funding: This research is supported by the Major Project of the National Social Science Fund of China: "Research on the Theoretical System, International Experience and Chinese Path of Evidence-based Social Science" (Project No. 19ZDA142);

Role of Funding Source: The funder had no further role in study design; in the collection, analysis and interpretation of data; in the writing of the report; and in the decision to submit the paper for publication.

Conflict of interests: The all authors declare that there is no conflict of interests.

Acknowledgements: None.

Authors' contributions: KLG, JWL, and JYL conceived and designed the study. NC, YFL and JYL selected the articles and extracted the data. KLG and JWL analyzed the data. JWL completed some background writing and KLG wrote the first draft of the manuscript. All authors were involved in the interpretation of data and contribution to the writing of the final manuscript.

\section{Abstract \\ Background}

Pharmacotherapies are widely used for smoking cessation. However, their effect on smoking cessation for people with alcohol dependence remains unclear.

\section{Objective}

This study aimed to explore the effects of pharmacotherapies on smoking cessation for people with alcohol dependence.

\section{Methods}

Five electronic databases were searched in January 2021 for randomized controlled trials (RCTs) reporting the use of pharmacotherapies to promote smoking cessation in people with alcohol dependence. The risk of bias was assessed using the Cochrane tool. RevMan version 5.3 was used to perform meta-analyses of the changes in smoking behavior, and the GRADE approach was used to assess the certainty of the evidence.

\section{Results}

Nine RCTs involving 908 smokers with alcohol dependence were identified. Eight RCTs were published in the United States, and one was from Canada. The risk of bias was rated as low in three studies and unclear in the remaining six. The results of the meta-analysis showed that, compared with the placebo group, Varenicline had a significant effect on short-term smoking cessation (three RCTs, OR $=6.27,95 \%$ CI: $[2.49,15.78], p<0.05$, very low certainty). Naltrexone had no significant effect on smoking cessation in short-term or long-term observations (three RCTs, OR $=0.99,95 \% \mathrm{CI}$ : [0.54, 1.81], $p=0.97$, moderate certainty), and Topiramate had no significant effect (two RCTs, OR $=1.56,95 \%$ CI: $[0.67,3.46], p>0.05$, low certainty). Only one trial reported that Bupropion had no effect on smoking cessation.

\section{Conclusion}


Varenicline may have a positive effect on smoking cessation in people with alcohol dependence. However, Naltrexone, Topiramate, and Bupropion seem to have no clear effect on increasing smoking abstinence among drinkers. The small number of studies and the low certainty of evidence indicate that caution is required in interpreting the results.

Keywords:Smoking Cessation, Alcohol Dependence, Pharmacotherapies, Systematic Review and Metaanalysis

\section{Introduction}

The health problems caused by smoking have become an important consideration for public health. According to a World Health Organization (WHO) report, smoking is a significant factor leading to cardiovascular and respiratory diseases and more than 20 different types or subtypes of cancer $[1,2]$. More than eight million people die from tobacco use each year, and most of these deaths occur in low- and middle-income countries[3]. However, tobacco use is not only harmful to smokers themselves, as second-hand smoke also affects non-smokers. According to WHO statistics, 1.2 million deaths each year are related to second-hand smoke exposure. More noteworthy is the impact of smoking, or second-hand smoke, on pregnant women and children. Each year, 65,000 children die from diseases related to second-hand smoke[4]. Smoking during pregnancy can also cause lifelong health problems for babies. [5] Therefore, various countries and organizations strive to find effective ways to help tobacco-dependent people quit smoking.

Alcohol use disorder (AUD) is one of the most common mental disorders in the world[6]. From 2012 to 2013 , the prevalence rates of 12-month and lifetime AUD among adults aged 18 years and older in the United States were $13.9 \%$ and $29.1 \%$, respectively, which accounted for approximately 33 million and 685 million people[7]. The corresponding Diagnostic and Statistical Manual of Mental Disorders (DSM-IV) rates increased by $12.7 \%$ and $43.6 \%$ for 12-month and lifetime AUD, respectively, over the year 2001[8]. For many people, AUD is a chronic recurrent remissive disorder[9]. Regardless of the intervention, at least $60 \%$ of people with AUD will relapse within six months of treatment and return to dangerous levels of alcohol consumption[10, 11]. The chronic addiction cycle of AUD is related to a variety of biological, psychological, and social characteristics[12]. Among them, smoking is closely related to drinking. Studies have shown that smoking is associated with excessive drinking, increased alcohol withdrawal symptoms, a higher relapse probability, or early relapse after treatment in individuals recovering from dangerous drinking habits[13, 14]. Therefore, the development of smoking cessation treatment programs for this population is important for their long-term health and recovery from addiction.

For people with alcohol dependence or heavy alcohol use, smoking cessation drugs have also been shown to reduce alcohol consumption and cravings[15]. The drugs currently approved by the United States Food and Drug Administration for the treatment of alcohol dependence (such as acetamide, naltrexone, or disulfiram) have a small to moderate effect on drinking[16, 17] but may not be as beneficial for smoking cessation[18]. Nicotine replacement therapy (NRT), bupropion, and varenicline are widely available prescriptions for smoking cessation. In the United States and the European Union, these drugs are licensed as first-line treatments for adjuvant smoking cessation treatment and are widely recommended in many national guidelines[19]. There are also studies[20] testing the effectiveness and safety of these drugs for treating people with alcohol dependence who are trying to quit smoking.

The purpose of this systematic review and meta-analysis was to assess the effectiveness and safety risks of drug therapies for smoking cessation in patients with alcohol dependence. The outcomes will help to provide relevant information for alcohol addicts, clinicians, and policymakers.

\section{Methods}

\section{Search strategy}

Online databases including PubMed, Cochrane Library, Web of Science, Embase, and Google Scholar were searched from inception to January 2021 to identify relevant studies. In addition, supplementary searches were conducted through the WHO International Clinical Trials Registry Platform (ICTRP) search portal and 
grey literature. The main search strategies were as follows: (smok* OR cigarette OR tobacco OR nicotine) AND (Alcohol* OR drink) AND (cessation OR quit* OR abstinence OR stop*) AND (drug OR medicine OR pharmaco*) AND ("random*" OR "blind*" OR "single-blind*" OR "double-blind*" OR "treble-blind*" OR "triple-blind*").

\section{Inclusion and exclusion criteria}

Only randomized controlled trials (RCTs) were included. The inclusion criteria were as follows: 1) Population: smokers with alcohol dependence; 2) Intervention: the experimental group accepted pharmacotherapies and the control group received a placebo; 3) Outcomes: only the indicators related to smoking were included, such as smoking cessation rate and changes in the number of cigarettes per day.

Studies that were duplicate reports or with insufficient data, such as protocols, conference proceedings, or abstracts, were excluded. Studies written in languages other than English were not included.

\section{Study selection and data extraction}

Two reviewers independently conducted screening and data extraction, with any differences resolved by consultation with a third reviewer. After removing duplicate articles, two reviewers screened the titles and abstracts according to the inclusion criteria and then determined the final studies for inclusion by reading the full text.

A predesigned table was used to collate the extracted data. One reviewer independently extracted data, and an expert verified the content. The data that were extracted from each publication included: (1) details of the study, such as the year of publication, study design, name of the first author, and country (2) characteristics of the population, such as their age and cigarettes smoked per day; (3) details of the intervention, such as the drug name, dose, and duration of treatment); (4) treatment outcomes, including the number of people who successfully quit smoking and any adverse reactions.

\section{Risk of bias assessment}

Two reviewers assessed the risk of bias of the included studies according to the Cochrane Risk of Bias (ROB) tool (Cochrane Handbook Version 5.1.0)[21, 22]. The ROB tool included seven domains: random sequence generation, allocation concealment, blinding of participants and personnel, blinding of outcome assessment, incomplete outcome data, selective reporting, and other biases. Each domain was judged as either yes, no, or unclear according to the information provided in the studies[23]. If all the domains were rated yes, the study was judged to be at low risk of bias, and if one or more domains were rated no, the study was judged to be at high risk of bias. Otherwise, the study was judged to have an unclear risk of bias. Disagreements in bias assessment were resolved by discussions among the two reviewers and, if necessary, through consultation with a third reviewer[24].

\section{Data analysis}

The meta-analysis was performed using RevMan version 5.3 software[25]. For continuous variables, data were synthesized using the mean difference (MD) with a $95 \%$ confidence interval (CI) or standardized mean difference (SMD). An odds ratio (OR) or risk ratio (RR) was used to analyze discontinuous variables. The Chi-squared test and $I$-squared $\left(I^{2}\right)$ tests were used to assess for statistical heterogeneity. High heterogeneity in the results was identified if the tests were statistically significant $(\mathrm{p}<0.05)$ and the $I^{2}>50 \%$, in which case a random-effects model was selected. Otherwise, a fixed-effects model was used. Sensitivity analysis was conducted by removing individual studies or eliminating studies with a high risk of bias. If necessary, a subgroup analysis was conducted according to the different intervention conditions (dose, duration, and population) [26].

\section{Certainty assessment}

The Grades of Recommendation, Assessment, Development, and Evaluation (GRADE) system ${ }^{11}$ were used to assess the certainty (quality) of evidence associated with specific outcomes and in constructing a findings 
summary table. In the process of evaluating the quality of evidence for RCTs, five downgrade factors, including the study limitations, consistency of effect, imprecision, indirectness, and publication bias, were used to estimate the certainty of the outcomes ${ }^{12}$. The outcomes were degraded from a high-quality level for each degrading factor, rated as either not serious, serious, or very serious.[27, 28] Depending on the severity, outcomes were considered to degrade one (serious) or two levels (very serious), with the final level of evidence quality considered as either high, moderate, low, or very low.

\section{Results}

\section{Study selection}

A PRISMA diagram of the search results, including reasons for exclusion, is shown in figure 1. A total of 836 relevant records were identified; however, 243 trials were removed because of duplication. Titles and abstracts were screened for 593 studies, of which 556 were deemed to be unsuitable. The full texts of the remaining 37 articles were screened, and 25 trials were excluded. Finally, nine RCTs were included[29-37].

\section{Study characteristics}

As shown in Table 1, the included RCTs involved 908 smokers with alcohol dependence (450 in treatment groups and 458 in control groups). All studies were published between 2005 and 2020 in the United States (n $=8)$ and Canada $(\mathrm{n}=1)$. Most smokers were over 40 years old and smoked more than ten cigarettes per day. The treatment group in each RCT examined one of four drugs, including Varenicline $(\mathrm{n}=3)$, Naltrexone $(\mathrm{n}=3)$, Topiramate $(\mathrm{n}=2)$, Bupropion $(\mathrm{n}=1)$, and all RCTs gave a placebo to the control group. The duration of the measurement phase ranged from two weeks to six months, and the outcome indicator of all studies was the smoking cessation rate

\section{Risk of bias}

As shown in figure 2, three studies[30,31,35] had a low risk of bias. These studies used a random treatment allocation sequence generated by a computer, allocation concealment with envelopes, and a double-blind execution to ensure the quality of the research design. The remaining six studies had an unclear risk of bias due to insufficient information in the reports.

\section{Treatment effects and adverse events}

\section{Varenicline}

Three RCTs[31, 35, 37] involving 195 smokers reported the smoking cessation effects of Varenicline. The results of the subgroup analysis showed that in less than 12 weeks, Varenicline improved the smoking cessation rate of tobacco dependents compared with the placebo group ( $\mathrm{OR}=1.22,95 \%$ CI: $[3.44,57.07], p<0.001)$. However, when the treatment duration was longer than 12 weeks, the difference between the two groups was not statistically significant $(\mathrm{OR}=2.32,95 \% \mathrm{CI}:[0.62,8.70],=0.21)$. The overall effect size indicated that the intervention was effective ( $\mathrm{OR}=6.27,95 \% \mathrm{CI}$ : $[2.49,15.78], p<0.001$; very low certainty; Figure 3$)$.

\section{Naltrexone}

Three RCTs[30, 32, 33] involving 432 smokers reported the smoking cessation effects of Naltrexone. The results of the subgroup analysis showed that, for two studies after eight weeks of treatment, there were no statistically significant differences in smoking cessation rates between the treatment and control groups (OR $=0.99,95 \%$ CI: $[0.54,1.81], p=0.97)$, nor were there any significant differences in a single study after 2 , 12, 16, or 26 weeks (figure 4) of treatment. The overall effect size indicated Naltrexone did not significantly increase the smoking cessation rate of alcohol-dependent people.

\section{Topiramate and Bupropion}

Two RCTs[34, 36] involving 223 smokers reported the smoking cessation effects of Topiramate. The results of the meta-analysis showed that Topiramate had no obvious smoking cessation effects $(\mathrm{OR}=1.56,95 \%$ CI: $[0.67,3.64], p=0.31$; Figure 5). As for Bupropion, only one RCT[29] was included. The results of 
the original study showed that the difference between the two groups was not statistically significant in the fourth $(\mathrm{p}=0.28)$ or ninth $(p=0.44)$ week of treatment or after six months $(\mathrm{p}=0.28)$. Bupropion did not increase the smoking cessation rate.

Adverse events

A detailed analysis of the possible damaging effects to the body caused by these drugs was made based on the adverse reactions reported in the included studies. As shown in table 2 , the four drugs may cause side effects, including vomiting, nausea, diarrhea, headache, dry mouth, sweating, insomnia, and dizziness. The difference in adverse reactions between the drug treatment and placebo groups was tested based on the value of the odds ratios (OR), the $95 \%$ confidence intervals, and the p-value. With the exception of two studies[29] that reported Naltrexone could cause more adverse events, the other studies all showed that there were no significant differences in the occurrence of adverse events between the treatment and control groups.

\section{Certainty of evidence}

The certainty of the evidence for each drug is shown in table 3. Varenicline was downgraded by three levels in total due to high heterogeneity (inconsistency) and wide confidence intervals (imprecision), and as such it was rated as having very low evidence. Naltrexone was downgraded by one level due to an unclear risk of bias, and the evidence for its effect was considered moderate. Topiramate was downgraded by two levels due to the small number of studies and high heterogeneity (inconsistency), such that the evidence was rated as low. Bupropion was downgraded by two levels due to a small number of studies and wide confidence intervals (imprecision), resulting in a low evidence rating.

\section{Discussion}

\section{Summary of the main results}

From the three studies on Varenicline, it was found that although it has an effect in the short term, it has no long-term effects (more than 12 weeks). However, the generalizability of the results is limited due to the small sample sizes and the wide confidence intervals of these studies. Therefore, the specific effects of Varenicline remain unclear, and the conclusions should be interpreted prudently.

There were three studies on Naltrexone, and the meta-analysis showed that it had no positive effect on smokers. It is worth pointing out that one of the studies of Naltrexone explored its effectiveness for smoking cessation in light drinkers and heavy drinkers. According to the original study's explanation, Naltrexone was ineffective for light drinkers but effective for heavy drinkers. However, the present analysis of the $95 \%$ confidence intervals showed that Naltrexone had no smoking cessation effect on either type of drinker.

As for Topiramate and Bupropion, the result indicated that neither had a good effect on smoking cessation. However, the smoking cessation effects of these two drugs have been explored in very few studies. As such, the conclusions may change with further studies in the future.

The results showed that there was no significant difference in the occurrence of adverse events between the drug group and the placebo group in general (Table 2). The symptoms occurred equally in people who did or did not receive the drug treatment, so these drugs are considered safe to use. However, clinicians and researchers should also be alert to people who may overreact to these drugs. If a serious adverse reaction occurs, the individual should terminate their participation in the trial.

\section{Comparisons with other studies or reviews}

This systematic review is different from other studies in that varying degrees of alcohol dependence were considered among smokers. This study found that the same drugs that have been used to explore smoking cessation treatments have also been used for treating other types of addiction, such as those who are dependent on alcohol. At the same time, many studies have paid attention to the smoking cessation effects of these drugs on different populations of smokers, such as pregnant women and people with schizophrenia. For example, Kishi et al.[38] conducted a systematic review to explore the effect of Varenicline on smoking 
cessation, and found that Varenicline was not superior to placebo for promoting smoking cessation in people with schizophrenia. Meanwhile, Oon-Arom[39] separately synthesized the evidence for Varenicline on alcoholism. These two studies show that Varenicline is a relatively important drug in the treatment of addictive behaviors. Moreover, Siskind[40] conducted a network meta-analysis to explore smoking cessation effects among people with schizophrenia spectrum disorders and pointed out that Varenicline might be superior to Bupropion. As for other drugs, Lotfy[41] and David[42] synthesized the effects of Topiramate and Naltrexone on smoking cessation, respectively, with both studies indicating that these drugs have no beneficial short-term or long-term effects on smoking abstinence. From these systematic reviews and meta-analyses, it can be seen that the populations studied were general smokers or smokers with schizophrenia. However, this study has found that some RCTs reported the smoking cessation effects of these drugs on alcohol-dependent people, but to date, there has been no relevant synthesis of this evidence.

\section{Quality assessment}

This study used the method in the Cochrane Handbook to assess the risk of bias of the included studies because the quality of the study has an important impact on the credibility of the results. A high-quality study design can generate more reliable evidence[43, 44]. Three of the nine included studies had a low risk of bias, while the others were unclear. Although there were no studies with a high risk of bias, four RCTs with an unclear risk of bias were identified due to reporting ambiguous details, especially for blinding and random sequence generation procedures. Alternatively, the three RCTs with a low risk of bias explained their procedures for generating random sequences using computer software or random number tables and reported blinded participants or researchers. These important methodological designs improved the validity and reliability of their results. However, some studies did not mention the details of the methodological design and how the trial was implemented and can, therefore, only be judged as having an unclear risk of bias. There is no doubt that an unclear risk of bias has the potential to be high risk, which reduces the reliability of the evidence. Therefore, randomized controlled design trials should emphasize reporting study design features such as randomization and blinding.

As for the certainty of the evidence, the GRADE results showed that Varenicline was very low, Naltrexone was moderate, and Topiramate and Bupropion were low. Considering that all the included studies were RCTs, only five degradation factors were considered. Among these factors, high heterogeneity (inconsistency), wide confidence intervals (imprecision), and low quality or a small number of included studies reduced the level of evidence. Especially for Varenicline and Topiramate, their high heterogeneity reduced the certainty of the evidence. The extent of the downgrade was based on the degree of heterogeneity, such that if heterogeneity was less than $50 \%$, the study was downgraded one level, otherwise downgrading by two levels was considered. The number of studies was also assessed as it would have an impact on the certainty of the evidence. The drugs in this systematic review only had a few studies each, especially for Topiramate and Bupropion, and as such, the level of evidence was reduced due to the small number of studies.

\section{Implications for future research}

Numerous clinical trials and systematic reviews have shown that drugs are routinely used to promote smoking cessation. Such drugs generally have the characteristics of treating addictive behaviors. That is, they have a positive or negative effect on the human nervous system. Based on the present research and other existing evidence, the following suggestions for future research can be made. The first pertains to the definition of the participants' characteristics. Populations with diverse characteristics, such as differences in age, sex, and health conditions, may have different treatment outcomes from the same experimental design. Smokers with different characteristics may foreseeably have different responses to the same drugs, which has the potential to confound the quality of research evidence. Therefore, future research should consider the potential impact of population characteristics on trials.

Another future research direction is adverse reactions to drug use. The effectiveness of drug treatments on the smoking cessation rate should not be the only focus, but the safety risks of the drugs for different populations should also be emphasized. Even if a certain drug has a positive effect on smoking cessation, it 
should not be considered as a treatment option if it has obvious detrimental effects on the body. At the same time, the dosage, frequency, and length of use of the drug should also be considered. Finding an appropriate drug intake rate not only eliminates possible harm to the body but also clarifies the intervention's effects. Therefore, it is necessary to make an accurate assessment of the dosage and safety risks of drugs in future research.

\section{Limitations}

There are some limitations in the present review. Firstly, although a search strategy was formulated and five databases were searched, there is a possibility of missing studies that meet the inclusion criteria. A supplementary search of grey literature databases and reference lists was conducted to reduce the risk of missing relevant studies. Secondly, the current conclusions may change with the publication of new results. Therefore, an update for this research should be performed within two years.

\section{Conclusion}

Based on the analysis of the studies included in this systematic review and meta-analysis, Varenicline may have a positive effect on smoking cessation in people with alcohol dependence in the short-term. However, Naltrexone, Topiramate, and Bupropion appear to have no clear effect on increasing smoking abstinence among drinkers. In particular, the small number of studies and low certainty of evidence have increased the uncertainty of the results. Therefore, in order to produce long-term and extensive evidence, more studies are needed to support these conclusions.

\section{References}

1. Park MS, Chung KY, Kim KD, Yang WI, Chung JH, Kim YS et al. Prognosis of thymic epithelial tumors according to the new World Health Organization histologic classification. The Annals of thoracic surgery. 2004;78(3):992-7; discussion 7-8. doi:10.1016/j.athoracsur.2004.03.097.

2. Harizi C, El-Awa F, Ghedira H, Audera-Lopez C, Fakhfakh R. Implementation of the WHO Framework Convention on Tobacco Control in Tunisia: Progress and challenges. Tobacco prevention \& cessation. 2020;6:72. doi: $10.18332 / \mathrm{tpc} / 130476$.

3. WHO. Leading cause of death, illness and impoverishment. 2020.https://www.who.int/news-room/factsheets/detail/tobacco .

4. Suliankatchi Abdulkader R, Sinha DN, Jeyashree K, Rath R, Gupta PC, Kannan S et al. Trends in tobacco consumption in India 1987-2016: impact of the World Health Organization Framework Convention on Tobacco Control. Int J Public Health. 2019;64(6):841-51. doi:10.1007/s00038-019-01252-x.

5. WHO. Tobacco smoking. 2018.https://apps.who.int/gho/data/node.sdg.3-a-viz?lang=en .

6. Rehm J, Anderson P, Barry J, Dimitrov P, Elekes Z, Feijão F et al. Prevalence of and potential influencing factors for alcohol dependence in Europe. European addiction research. 2015;21(1):6-18. doi: $10.1159 / 000365284$.

7. Grant BF, Goldstein RB, Saha TD, Chou SP, Jung J, Zhang H et al. Epidemiology of DSM-5 Alcohol Use Disorder: Results From the National Epidemiologic Survey on Alcohol and Related Conditions III. JAMA Psychiatry. 2015;72(8):757-66. doi:10.1001/jamapsychiatry.2015.0584.

8. Hasin DS, Stinson FS, Ogburn E, Grant BF. Prevalence, correlates, disability, and comorbidity of DSM-IV alcohol abuse and dependence in the United States: results from the National Epidemiologic Survey on Alcohol and Related Conditions. Archives of general psychiatry. 2007;64(7):830-42. doi:10.1001/archpsyc.64.7.830.

9. Witkiewitz K. Predictors of heavy drinking during and following treatment. Psychology of addictive behaviors : journal of the Society of Psychologists in Addictive Behaviors. 2011;25(3):426-38. doi:10.1037/a0022889. 
10. Durazzo TC, Meyerhoff DJ. Psychiatric, Demographic, and Brain Morphological Predictors of Relapse After Treatment for an Alcohol Use Disorder. Alcoholism, clinical and experimental research. 2017;41(1):10716. doi:10.1111/acer.13267.

11. Kirshenbaum AP, Olsen DM, Bickel WK. A quantitative review of the ubiquitous relapse curve. Journal of substance abuse treatment. 2009;36(1):8-17. doi:10.1016/j.jsat.2008.04.001.

12. Seo S, Mohr J, Beck A, Wüstenberg T, Heinz A, Obermayer K. Predicting the future relapse of alcoholdependent patients from structural and functional brain images. Addiction biology. 2015;20(6):1042-55. doi:10.1111/adb.12302.

13. Nguyen LC, Durazzo TC, Dwyer CL, Rauch AA, Humphreys K, Williams LM et al. Predicting relapse after alcohol use disorder treatment in a high-risk cohort: The roles of anhedonia and smoking. J Psychiatr Res. 2020;126:1-7. doi:10.1016/j.jpsychires.2020.04.003.

14. Chiappetta V, García-Rodríguez O, Jin CJ, Secades-Villa R, Blanco C. Predictors of quit attempts and successful quit attempts among individuals with alcohol use disorders in a nationally representative sample. Drug Alcohol Depend. 2014;141:138-44. doi:10.1016/j.drugalcdep.2014.05.019.

15. McKee SA, Harrison EL, O'Malley SS, Krishnan-Sarin S, Shi J, Tetrault JM et al. Varenicline reduces alcohol self-administration in heavy-drinking smokers. Biological psychiatry. 2009;66(2):185-90. doi:10.1016/j.biopsych.2009.01.029.

16. Skinner MD, Lahmek P, Pham H, Aubin H-J. Disulfiram Efficacy in the Treatment of Alcohol Dependence: A Meta-Analysis. Plos One. 2014;9(2). doi:10.1371/journal.pone.0087366.

17. Streeton C, Whelan G. Naltrexone, a relapse prevention maintenance treatment of alcohol dependence: A meta-analysis of randomized controlled trials. Alcohol and Alcoholism. 2001;36(6):544-52. doi:10.1093/alcalc/36.6.544.

18. Baltieri DA, Daro FR, Ribeiro PL, de Andrade AG. Effects of topiramate or naltrexone on tobacco use among male alcohol-dependent outpatients. Drug and Alcohol Dependence. 2009;105(1-2):33-41. doi:10.1016/j.drugalcdep.2009.05.025.

19. A clinical practice guideline for treating tobacco use and dependence: 2008 update. A U.S. Public Health Service report. American journal of preventive medicine. 2008;35(2):158-76. doi:10.1016/j.amepre.2008.04.009.

20. Zawertailo L, Ivanova A, Ng G, Le Foll B, Selby P. Safety and Efficacy of Varenicline for Smoking Cessation in Alcohol-Dependent Smokers in Concurrent Treatment for Alcohol Use Disorder: A Pilot, Randomized Placebo-Controlled Trial. J Clin Psychopharmacol. 2020;40(2):130-6. doi:10.1097/JCP.0000000000001172.

21. Cumpston M, Li T, Page MJ, Chandler J, Welch VA, Higgins JP et al. Updated guidance for trusted systematic reviews: a new edition of the Cochrane Handbook for Systematic Reviews of Interventions. The Cochrane database of systematic reviews. 2019;10:Ed000142. doi:10.1002/14651858.Ed000142.

22. Higgins JP, Altman DG, Gøtzsche PC, Jüni P, Moher D, Oxman AD et al. The Cochrane Collaboration's tool for assessing risk of bias in randomised trials. BMJ (Clinical research ed). 2011;343:d5928. doi:10.1136/bmj.d5928.

23. Bei P, Long G, Yang-Qin X, Ya-Jing C, Cai-Yun G, Xue H et al. Exercise training modalities in patients with type 2 diabetes mellitus: a systematic review and network meta-analysis. International Journal of Behavioral Nutrition and Physical Activity. 2018;15(1):72-.

24. Xiu-xia L, Ya Z, Yao-long C, Ke-hu Y, Zong-jiu Z. The reporting characteristics and methodological quality of Cochrane reviews about health policy research. Health policy (Amsterdam, Netherlands). 2015;119(4):503-10. doi:10.1016/j.healthpol.2014.09.002. 
25. Cochrane. RevMan 5-The desktop version of the software used for editing reviews. 2020.https://community.cochrane.org/help/tools-and-software/revman-5 .

26. Jia WQ, Tian JH, Yang KH, Ma B, Liu YL, Zhang P et al. Open versus Laparoscopic Pyloromyotomy for Pyloric Stenosis: A Meta-analysis of Randomized Controlled Trials. European Journal of Pediatric Surgery. 2010.

27. Guyatt G, Oxman AD, Akl EA, Kunz R, Vist G, Brozek J et al. GRADE guidelines: 1. IntroductionGRADE evidence profiles and summary of findings tables. Journal of clinical epidemiology. 2011;64(4):383-94. doi:10.1016/j.jclinepi.2010.04.026.

28. Guyatt GH, Oxman AD, Vist G, Kunz R, Brozek J, Alonso-Coello P et al. GRADE guidelines: 4. Rating the quality of evidence-study limitations (risk of bias). Journal of clinical epidemiology. 2011;64(4):407-15. doi:10.1016/j.jclinepi.2010.07.017.

29. Grant KM, Kelley SS, Smith LM, Agrawal S, Meyer JR, Romberger DJ. Bupropion and nicotine patch as smoking cessation aids in alcoholics. Alcohol (Fayetteville, NY). 2007;41(5):381-91. doi:10.1016/j.alcohol.2007.03.011.

30. Kahler CW, Cioe PA, Tzilos GK, Spillane NS, Leggio L, Ramsey SE et al. A Double-Blind Randomized Placebo-Controlled Trial of Oral Naltrexone for Heavy-Drinking Smokers Seeking Smoking Cessation Treatment. Alcoholism: Clinical and Experimental Research. 2017;41(6):1201-11. doi:10.1111/acer.13396.

31. O'Malley SS, Zweben A, Fucito LM, Wu R, Piepmeier ME, Ockert DM et al. Effect of Varenicline Combined With Medical Management on Alcohol Use Disorder With Comorbid Cigarette Smoking. JAMA psychiatry. 2018;75(2). doi:10.1001/jamapsychiatry.2017.3544.

32. King A, Cao D, Vanier C, Wilcox T. Naltrexone Decreases Heavy Drinking Rates in Smoking Cessation Treatment: An Exploratory Study. Alcoholism: Clinical and Experimental Research. 2009;33(6):1044-50. doi:10.1111/j.1530-0277.2009.00925.x.

33. Fridberg DJ, Cao D, Grant JE, King AC. Naltrexone Improves Quit Rates, Attenuates Smoking Urge, and Reduces Alcohol Use in Heavy Drinking Smokers Attempting to Quit Smoking. Alcoholism: Clinical and Experimental Research. 2014;38(10):2622-9. doi:10.1111/acer.12513.

34. Anthenelli RM, Heffner JL, Wong E, Tibbs J, Russell K, Isgro M et al. A Randomized Trial Evaluating Whether Topiramate Aids Smoking Cessation and Prevents Alcohol Relapse in Recovering Alcohol-Dependent Men. Alcoholism: Clinical and Experimental Research. 2017;41(1):197-206. doi:10.1111/acer.13279.

35. Zawertailo L, Ivanova A, Ng G, Le Foll B, Selby P. Safety and Efficacy of Varenicline for Smoking Cessation in Alcohol-Dependent Smokers in Concurrent Treatment for Alcohol Use Disorder. Journal of clinical psychopharmacology. 2020;40(2):130-6. doi:10.1097/jcp.0000000000001172.

36. Johnson BA, Ait-Daoud N, Akhtar FZ, Javors MA. Use of oral topiramate to promote smoking abstinence among alcohol-dependent smokers: a randomized controlled trial. Archives of internal medicine. 2005;165(14):1600-5. doi:10.1001/archinte.165.14.1600.

37. Hurt RT, Ebbert JO, Croghan IT, Schroeder DR, Hurt RD, Hays JT. Varenicline for tobacco-dependence treatment in alcohol-dependent smokers: A randomized controlled trial. Drug and alcohol dependence. 2018;184:12-7. doi:10.1016/j.drugalcdep.2017.11.017.

38. Kishi T, Iwata N. Varenicline for smoking cessation in people with schizophrenia: systematic review and meta-analysis. European archives of psychiatry and clinical neuroscience. 2015;265(3):259-68. doi:10.1007/s00406-014-0551-3.

39. Oon-Arom A, Likhitsathain S, Srisurapanont M. Efficacy and acceptability of varenicline for alcoholism: A systematic review and meta-analysis of randomized-controlled trials. Drug and alcohol dependence. 
2019;205:107631. doi:10.1016/j.drugalcdep.2019.107631.

40. Siskind DJ, Wu BT, Wong TT, Firth J, Kisely S. Pharmacological interventions for smoking cessation among people with schizophrenia spectrum disorders: a systematic review, meta-analysis, and network metaanalysis. The lancet Psychiatry. 2020;7(9):762-74. doi:10.1016/s2215-0366(20)30261-3.

41. Lotfy N, Elsawah H, Hassan M. Topiramate for smoking cessation: Systematic review and meta-analysis. Tobacco prevention \& cessation. 2020;6:14. doi:10.18332/tpc/115167.

42. David SP, Chu IM, Lancaster T, Stead LF, Evins AE, Prochaska JJ. Systematic review and metaanalysis of opioid antagonists for smoking cessation. BMJ open. 2014;4(3):e004393. doi:10.1136/bmjopen2013-004393.

43. Tian J, Zhang J, Ge L, Yang K, Song F. The methodological and reporting quality of systematic reviews from China and the USA are similar. Journal of clinical epidemiology. 2017;85:50-8. doi:10.1016/j.jclinepi.2016.12.004.

44. Yao L, Sun R, Chen YL, Wang Q, Wei D, Wang X et al. The quality of evidence in Chinese meta-analyses needs to be improved. Journal of clinical epidemiology. 2016;74:73-9. doi:10.1016/j.jclinepi.2016.01.003.

\section{Hosted file}

Figure.docx available at https://authorea.com/users/396700/articles/509811-the-effects-ofpharmacological-interventions-on-smoking-cessation-in-people-with-alcohol-dependence-asystematic-review-and-meta-analysis-of-nine-randomized-controlled-trials

\section{Hosted file}

Table.docx available at https://authorea.com/users/396700/articles/509811-the-effects-ofpharmacological-interventions-on-smoking-cessation-in-people-with-alcohol-dependence-asystematic-review-and-meta-analysis-of-nine-randomized-controlled-trials 